\title{
GGA + U Approximation: An Improved Density Functional Theory of Optical Properties of $\mathrm{CaH}_{2}$
}

\author{
Uko Ofe ${ }^{1}$, Anthony Lordson Amana2 ${ }^{2}$, Nsed A. Akonjom ${ }^{3}$ \\ ${ }^{1}$ Department of Pure and Applied Physics, Veritas University, Abuja, Nigeria \\ ${ }^{2}$ Department of Electronic and Computer Engineering, Veritas University, Abuja, Nigeria \\ ${ }^{3}$ Department of Physics, Cross-River State University, Calabar, Nigeria \\ Email: Ukoofe@gmail.com, amanaa@veritas.edu.ng, nakonjom@gmail.com
}

How to cite this paper: Ofe, U., Amana, A.L. and Akonjom, N.A. (2021) GGA + U Approximation: An Improved Density Functional Theory of Optical Properties of $\mathrm{CaH}_{2}$. Open Access Library Journal, 8: e7293.

https://doi.org/10.4236/oalib.1107293

Received: March 8, 2021

Accepted: September 20, 2021

Published: September 23, 2021

Copyright $\odot 2021$ by author(s) and Open Access Library Inc.

This work is licensed under the Creative Commons Attribution International License (CC BY 4.0).

http://creativecommons.org/licenses/by/4.0/

\begin{abstract}
In this paper, the optical properties of $\mathrm{CaH}_{2}$, in orthorhombic structure, with space group, $P_{n m a}$, in crystal system $\left(\mathrm{Co}_{2} \mathrm{Si}\right.$-type $)$ have been investigated carefully. The theoretical milieu of the correlation between the dielectric function with other optical constants has been investigated. The real and the imaginary parts of the dielectric function have besides, been inspected accurately. The outcome of the exchange correlation potentials implemented (GGA and GGA + U) to the absorption peaks and edges of this insulator $\left(\mathrm{CaH}_{2}\right)$, have also, been determined. It was noticed that the application of GGA + U results in the shift of the first absorption peak caused by the conduction band (imaginary part), thus resulting in the band correlation.
\end{abstract}

\section{Subject Areas}

Applied Physics, Function Theory, Solid Mechanics

\section{Keywords}

Dielectric Function, Optical Properties, GGA + U and GGA Approximations

\section{Introduction}

The electronic band structure and the structural property (Lattice constant) of $\mathrm{CaH}_{2}$ in orthorhombic structure, with space group, $P_{n m a}$, in crystal system ( $\mathrm{Co}_{2} \mathrm{Si}$-type) have been computed, using the density functional theory. The Kohn-Sham equation was solved, using the full-potential linearized augmented plane wave (FP-LAPW) [1]. Generalized Gradient Approximation (GGA) and GGA + U approximation were employed as exchange correlation potentials, with WIEN 2K Code. Designated method of the observables was expounded in 
[2]. The initial crystal structure of $\mathrm{CaH}_{2}$ was studied by Zintl and Harder [3] and Gridani and Mouhtadi (2000) employed the Hatree-Fock ab nitio method to investigate same properties of $\mathrm{CaH}_{2}$ solid system, and found it to be a strongly ionic insulator. Up-to-date, structural, electronic and optical properties of $\mathrm{MgH}_{2}$ and $\mathrm{CaH}_{2}$ have been studied, using GGA under [4]; their calculated band structure depicted that $\mathrm{MgH}_{2}$ has an insulating nature, while $\mathrm{CaH}_{2}$ exhibited semi-conducting behaviour, which is antithetical to the findings of the above researchers.

In this current work, the optical properties of $\mathrm{CaH}_{2}$ are to be examined, using full-potential linearized augmented plane (FP-LAPW), GGA and GGA + U approximations, with WIEN2K codes in the frame work of density functional theory (DFT).

\section{Theoretical Consideration}

\section{Dielectric Function}

The dielectric function describes what an electric field such as oscillating light wave does to a material. The Dielectric function is a three-dimensional tensor which depends on the symmetry of crystal, and can be calculated directly from the Kohn-Sham energy eigenvalues, $\varepsilon_{k}$. In the Random Phase Approximation (RPA), the function, $\varepsilon_{i j}(\omega)$, can be expressed as [5]

$$
\begin{aligned}
\varepsilon_{i j}= & \delta_{i j}-\frac{1}{v \omega^{2}} \sum_{n, k}\left(\frac{-\delta F(\varepsilon)}{\delta \varepsilon_{\varepsilon_{n, k}}}\right) P_{i ; n, n, k} P_{j ; n, n, k} \\
& -\frac{4 \pi}{v \omega_{2}} \sum \frac{P_{i ; c, v, k} P_{j ; c, v, k}}{\left(\varepsilon_{c, k}-\varepsilon_{v, k}\right)\left(\varepsilon_{c, k}-\varepsilon_{v, k}\right)^{2}}
\end{aligned}
$$

where $V$ is a unit cell Volume, $P_{n, m, k}$ are momentum matrix elements between the bands $n$ and $m$, for the point $K$ of the crystal. $F(\varepsilon)$ is a Fermi-Dirac distribution function:

$$
F(\varepsilon)=\frac{1}{\exp \left(\frac{\varepsilon-\varepsilon_{F}}{K_{B} T}\right)+1}
$$

where $\varepsilon_{F}$ is a Fermi level.

\section{Optical Properties}

\subsection{Imaginary and Real Parts of the Dielectric Function}

The imaginary part of the dielectric function is calculated in order to understand the optical properties of $\mathrm{CaH}_{2}$. The study of the optical properties is pivotal for understanding of the electronic structure of materials [6]. These can be obtained from the complex dielectric function $\varepsilon(\omega)$, which is in defined [6] as

$$
\varepsilon(\omega)=\varepsilon_{1}(\omega)+i \varepsilon_{2}(\omega)
$$

The imaginary part $\varepsilon_{2}(\omega)$ of the dielectric function can be calculated using 
momentum matrix elements [7]. The corresponding eigen-function of each of the occupied and unoccupied state contributes to the matrix elements [8]. The real parts $\varepsilon_{1}(\omega)$ of the dielectric function can be derived from the imaginary part $\varepsilon_{2}(\omega)$ by Krong-Kramers relationship [8].

At this point, it is apropos to mention that the imaginary part of the dielectric function, also, is indicative of real transfers between the occupied and unoccupied states, thus the imaginary part then, controls the attenuation, while the real part explains refraction. In other words, the real part marks scattering and loss in optical processes.

\subsection{Refractive Index and Extinction Coefficient}

The refractive index determines how much light is bent or refracted, when entering a material. The refractive and extinction coefficients are intrinsically related, for they are derived from the same physical process. The refractive index and the extinction coefficients are tensors, and are expressed as

$$
n_{i i}(\omega)=\sqrt{\frac{\left|\varepsilon_{i i}(\omega)\right|+\operatorname{Re} \varepsilon_{i i}(\omega)}{2}}
$$

and

$$
k_{i i}(\omega)=\sqrt{\frac{\left|\varepsilon_{i i}(\omega)\right|-\operatorname{Re} \varepsilon_{i i}(\omega)}{2}}
$$

where $n_{i i}(\omega)$ is the refractive index, and $k_{i i}(\omega)$ is the extinction coefficient.

\subsection{Reflectivity and Absorption Coefficient}

In optical experiments, $n_{i i}(\omega)$ and $k_{i i}(\omega)$ cannot be measured explicitly. The measurable quantities are reflectivity $R_{i i}(\omega)$, and the absorption coefficient $A_{i i}(\omega)$. It can be shown in Literature on electromagnetism that these quantities can be expressed as [5]:

$$
R_{i i}(\omega)=\frac{\left(n_{i i}(\omega)-1\right)^{2}+k_{i i}^{2}(\omega)}{\left(n_{i i}(\omega)+1\right)^{2}+k_{i i}^{2}(\omega)}
$$

and

$$
A_{i i}(\omega)=\frac{2 \omega k_{i i}(\omega)}{c}
$$

\section{Computational Methods}

To eschew verbosity, the detailed computational method is presented in the electronic and structural properties of $\mathrm{CaH}_{2}$, using GGA and GGA + U approximations, with WIEN2K codes [1].

\section{Results and Discussion}

\section{Absorption Edge for GGA and GGA + U Functional}

The calculated dielectric function for $\mathrm{CaH}_{2}$ is portrayed in Figure 1. 


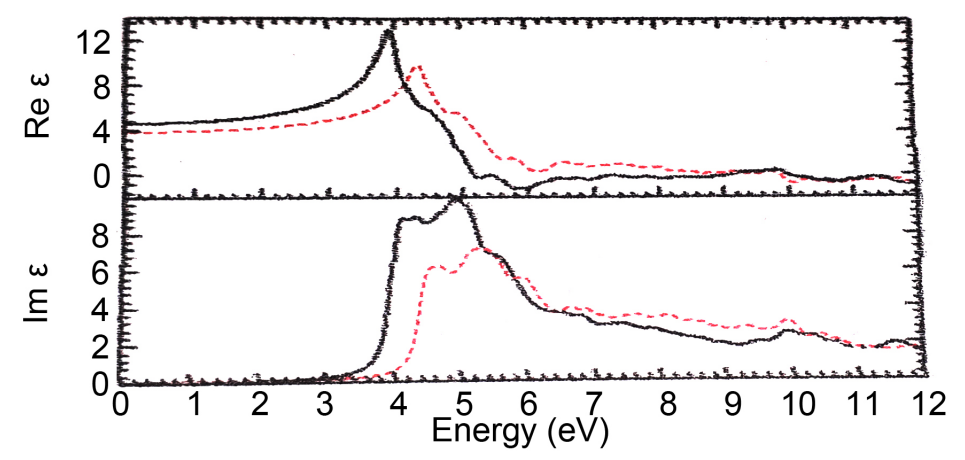

Figure 1. Real and imaginary parts of the dielectric function of $\mathrm{CaH}_{2}$.

The black solid lines are for GGA-PBE and the dash line for GGA + U calculations. It was observed that the compound has one leading absorption peak at $5.6 \mathrm{eV}$ and one minor one at $4.5 \mathrm{eV}$. It was also noticed that implementation of the GGA + U functional induces the alteration in the first absorption peak caused by the shift in the conduction band.

\section{Conclusion}

The dielectric function of Sodium hydride $(\mathrm{NaH})$, which is the fundamental quantity that appertains to the electronic structure, and defines its optical properties, has been determined. It was observed that the hydrogen embedded in the compound, including the XC, GGA + U, applied changes its band gaps, thus making it more insulating.

\section{Acknowledgements}

This work was supported by the Department of Physics, Veritas University Abuja.

\section{Data Availability}

The data that support the findings of this study are available from the corresponding author upon reasonable request.

\section{Conflicts of Interest}

The authors declare no conflicts of interest.

\section{References}

[1] Uko, O., Michael, U.O. and Udoimuk, A.B. (2014) Electonic and Structural Properties of $\mathrm{CaH}_{2}$, Using GGA and GGA + U Approximations, with WIEN2k Codes. Innovative Space of Scientic Research Journals, 27, 252-262.

[2] Setten, V., Popa, V.A. and de Wijs, G.A.B. (2007) Electronic Structure and Optical Properties of Lightweight Metal Hydrides. Physics Review, B15, 35204. https://doi.org/10.1103/PhysRevB.75.035204

[3] Wu, H., Zhou, W., Udovic, T.J., Rush, J.J. and Yildirim, J.J. (2007) Structure and Vibrational Spectra of Calcium Hydroxide and Deuteride. Journal of Alloys and 
Compound, 436, 51-52. https://doi.org/10.1016/j.jallcom.2006.07.042

[4] El. Gridani, A. and Mohammed, E.M. (2000) Electronic and Structural Properties of $\mathrm{CaH}_{2}$ : An Nition Hatree-Fock Study. Chemical Physics, 252, 1-8. https://doi.org/10.1016/S0301-0104(99)00333-X

[5] Ambrosch-Daxl, C. and Sofo, J.O. (2006) Linear Properties of Solids within the Full-Potential Linearized Augmented Plane Wave Method. Physics, Institute for Computational and Data Sciences (ICDS), Material Research Institute (MRI).

[6] Mark, F. (2001) Optical Properties Od Solids. Oxford University Press, Oxford.

[7] Starace, A.F. and Siamak, S. (1982) Random-Phase Approximation to One-Body Transition Metal Element for Open-Shell Atoms. Physics Review, A25, 2135. https://doi.org/10.1103/PhysRevA.25.2135

[8] Nussenzveig, H.M. (1972) Casualty and Dispersion Relations. New York Academic, New York, 45. 